\title{
Evaluation of Power Indicators of the Automobile Engine
}

\author{
Hayder Abed Dhahad ${ }^{1}$, Wissam Hameed Alawee ${ }^{1}$, Andrii Marchenko ${ }^{2}$, Dmytro Klets ${ }^{3 *}$, Oleg Akimov ${ }^{2}$ \\ ${ }^{1}$ University of Technology (Baghdad, Iraq) \\ ${ }^{2}$ National Technical University "Kharkiv Polytechnic Institute” (Kharkiv, Ukraine) \\ ${ }^{3}$ Kharkiv National Automobile and Highway University (Kharkiv, Ukraine) \\ *Corresponding author E-mail: d.m.klets@gmail.com
}

\begin{abstract}
A method for estimating the power characteristics of the car engine in various driving regimes is proposed. It is determined that the volume of the free power (engine power reserve) allows the driver to implement the turning parameters and the time of its completion, which are set by the steering parameters, position and the speed of the steering wheels rotation. The engine power reserve is necessary to provide the required maneuverability indicators. The engine power during maneuvering is spent on overcoming the resistance to movement and on providing the impact controlled by the maneuver. The first component of the engine power can be called connected, and the second one can be called the free one or a controlled component. The received analytical expressions allow carrying out at the design stage a rational choice of capacity of the engine by the condition of maintenance of demanded properties of maneuverability. The developed method for constructing the refined acceleration characteristics of the engine makes it possible to determine the dynamic capabilities of the car, taking into account its design features and operating conditions.
\end{abstract}

Keywords: Automobile; Acceleration; Engine; Power; Test.

\section{Introduction}

Currently, there is an increase in engine power in relation to the total mass of the car (the specific power of cars). The noted growth can be explained by an increase in the maximum design speed of vehicles, improved comfort (an increase in the number of motor energy users, providing functions that are not directly related to the movement of cars).

To evaluate the power indicators developed by the engine during operation, high-speed, load, adjusting, and multiparametric characteristics are determined, as well as the characteristics of optimal control, idling and toxicity. Increasing the accuracy of the engine power rating will in turn make it possible to increase the accuracy of determining the indicators of the functional and technical efficiency of the car, as well as its dynamic properties.

\section{Analysis of the Literature Data and the Formulation of the Problem}

A number of works by domestic and foreign scientists deal with the determination of engine power parameters [1-3]. In patent [4] they propose a method for determining the engine power of the car, depending on the parameters of its movement, taking into account the operating conditions. The power is proposed to be determined after preliminary run-out, using accelerometers, by simultaneous measurement of all types of drag of the car, that is, the moments of resistance occurring in the vehicle transmission, as well as the total road and aerodynamic drag. This method requires high accuracy when processing the run-out parameters to eliminate errors. The authors of work [5] proposed algorithms for the experimental determination of the engine power of a mobile machine, which is implemented during the execution of technological operations, using the method of partial accelerations. These algorithms allow determining the realizable power of the engine when the mobile machine is operating with trailed or mounted implements and on the power take-off shaft. This allows, in turn, proceeding to the determination of the effective torque and the effective power of the wheeled car engine under operating conditions. To implement this method, it is necessary to differentiate the angular velocity and determine the effective torque with the use of the results of the previous run-out. Increasing the power of an automobile engine can affect its strength characteristics. In [6-7], the influence of the regimes of strengthening combined treatment as well as quenching on the change in the properties of the surface layer of steel parts was investigated. Optimization of energy consumption of engines is dealt with in works [8-10]. The energy optimization strategy for solving the energy management problem in hybrid electric vehicles with high power is formulated in work [8], and in work $[9,10]$ the energy costs of vehicles of individual fleets are investigated and their growth is forecasted. The schemes of onboard monitoring of the technical condition of power units of wheeled and tracked vehicles are analyzed in work [11]. The ecological analysis of simple and combined thermal and power internal combustion engines was carried out in works [12, 13].

The author of work [14] proposed a technique for carrying out the qualimetry of vehicles based on the kinetic energy used for the movement of cargo, taking into account the design features of the vehicle. However, this technique does not take into account the dynamic properties of cars, which are manifested during acceleration. Estimation of the potential dynamic properties of cars is possible by determining the acceleration characteristics of the engine [15], i.e. the dependence of power and torque on the angular velocity of the crankshaft. Thus, the problem of increasing the accuracy of estimating the power parameters of the automobile engine with the help of engine acceleration characteristics requires additional study. 


\section{Purpose and Objectives of the Study}

The purpose of the study is to develop a method for estimating the power ratings of the automobile engine in various driving regimes. To achieve this goal, it is necessary to solve the following tasks:

- determine the realizable engine power in driving;

- to develop a refined method for building the acceleration characteristics of the automobile engine;

- carry out an experimental evaluation of the automobile engine power characteristics.

\section{Determination of the Realizable Power of the Engine When the Car is Turning}

Additional power input to provide the turning of the car is an indicator of ease of steering, controllability and maneuverability of the car. The engine power reserve determines the dynamic properties (dynamism) of the car and is its qualimetric characteristic. In works $[5,15]$ the engine power reserve was associated with the acceleration (dynamics of acceleration) of the car.

When designing cars, it is necessary to create a reserve of engine power, not only to create the possibility of engine acceleration, but also for the possibility of making a turn. Both are different types of vehicle maneuver, which requires additional engine power.

The additional power of the engine is also used to ensure the stability of steady translational motion with a sharp increase in resistance to vehicle movement.

In work [15], they determined the tangential reactions on the driving wheels of the car during cornering. The dependence for determining the sum of the tangential reactions on the laterally rigid front and rear wheels has the form of

$$
\begin{gathered}
R_{K_{2}}+R_{K_{1}} \cdot \sec \bar{\alpha}=m_{a} \cdot\left\{\left[1+\frac{b^{2}+i_{Z}^{2}+f \cdot b \cdot h}{L^{2}} \cdot \operatorname{tg}^{2} \bar{\alpha}\right] \times\right. \\
\left.\times \frac{d \omega_{Z}}{d t} \cdot L \cdot \operatorname{ctg} \bar{\alpha}+V_{a}^{2} \cdot \frac{h}{L^{2}} \cdot \operatorname{tg}^{2} \bar{\alpha}\right\},
\end{gathered}
$$

where $R_{K_{2}} ; R_{K_{1}}$ are the tangential reactions of the road on the front and rear wheels of the car;

$\bar{\alpha}$ is the average angle of rotation of the steering wheels;

$L$ is the wheelbase of the car,

$b$ is the distance from the rear axle to the projection of the car center of mass on the plane passing through the axes of the front and rear wheels;

$i_{Z}$ is the radius of inertia of the vehicle relative to the vertical axis;

$h$ is the height of the center of mass;

$f$ is the coefficient of the rolling resistance of wheels;

$\omega_{Z} ; \frac{d \omega_{Z}}{d t}$ is the angular velocity and angular acceleration of the car in the plane of the road.

In work [4], they have clarified the distribution of normal reactions on the wheels of the front and rear axles, taking into account the action of torque moments. With due consideration of the results of the study [4], let's transform expression (1) in the form of

$$
\begin{gathered}
R_{K_{2}}+R_{K_{1}} \cdot \sec \bar{\alpha}=m_{a} \cdot\left\{\left[1+\frac{b^{2}+i_{Z}^{2}+f \cdot b \cdot\left(h-r_{d}\right)}{L^{2}} \cdot \operatorname{tg}^{2} \bar{\alpha}\right] \times\right. \\
\left.\times \frac{d \omega_{Z}}{d t} \cdot L \cdot \operatorname{ctg} \bar{\alpha}+V_{a}^{2} \cdot f \cdot \frac{h-r_{d}}{L^{2}} \cdot \operatorname{tg}^{2} \bar{\alpha}\right\},
\end{gathered}
$$

where $r_{d}$ is the dynamic radius of the wheels (adopted the same for all the wheels of the car).

Dependence (2) is universal and allows determining the total tangential reaction

$$
R_{K^{\prime}}=R_{K_{2}}+R_{K_{1}} \cong R_{K_{2}}+R_{K_{1}} \cdot \sec \bar{\alpha}
$$

for vehicles with an all-wheel drive, only to the rear or only to the front wheels. This relationship is valid in the case when the tangential reactions, together with the lateral ones, do not exceed the limiting forces on the front and rear axles, respectively, i.e.

$\sqrt{R_{K_{1}}^{2}+R_{l_{1}}^{2}} \leq P_{c f_{1}}=\varphi \cdot R_{Z_{1}}$

$\sqrt{R_{K_{2}}^{2}+R_{l_{2}}^{2}} \leq P_{c f_{2}}=\varphi \cdot R_{Z_{2}}$

where $R_{l_{1}} ; R_{l_{2}}$ are the total lateral forces on the wheels of the front and rear axles, which arise when the car turns;

$P_{c f_{1}} ; P_{c f_{2}}$ are the total limiting forces on the clutch on the wheels of the front and rear axles, respectively;

$\varphi$ is the coefficient of wheels traction with the road;

$R_{Z_{1}} ; R_{Z_{2}}$ are the total normal reactions on the wheels of the front and rear axles.

If conditions (9) and (10) are not fulfilled, the wheels of either the front or rear axle are laterally slid. The behavior of cars in this case will be the topic of a separate study.

Regardless of the drive scheme of the driving wheels of the car (full, front or rear drive), the relationship between the total tangential reaction $R_{K}$ and the total tractive force $P_{K}$ is determined by the following relationship

$P_{K}=R_{K}+P_{f}=P_{K}+m_{a} \cdot g \cdot f$,

where $P_{f}$ is the total rolling resistance of the vehicle;

$g$ is acceleration of gravity, $g=9.81 \mathrm{~m} / \mathrm{s}^{2}$.

In work [15], in determining the forces acting in the contact of the wheels with the road, the force of the aerodynamic resistance to the movement of the car was not taken into account, since it was assumed that the motion occurs at the speed of $V a \leq 60 \mathrm{~km} / \mathrm{h}$.

Applying the principle of superposition in mechanics, we'll determine the total tractive power of the car when cornering, taking into account the air resistance

$P_{w}=k \cdot F \cdot V_{a}^{2}$,

where $k \cdot F$ is the factor of air resistance (the coefficient of air resistance, $F$ is the frontal area of the car or the midsection). Substituting (2) and (7) into (6), we obtain

$$
\begin{aligned}
R_{K}=m_{a} \cdot[g & \cdot f+\frac{k \cdot F}{m_{a}} \cdot V_{a}^{2}+\frac{R^{2}+b^{2}+i_{Z}^{2}+f \cdot b \cdot\left(h-r_{d}\right)}{R} \times \\
& \left.\times \frac{d \omega_{Z}}{d t}+\omega_{Z}^{2} \cdot f \cdot\left(h-r_{d}\right)\right]
\end{aligned}
$$

where $R$ is the turning radius of the car; for cars with laterally rigid wheels

$$
R=L \cdot \operatorname{ctg} \bar{\alpha} .
$$

The engine power, expended on the movement of the car when turning 


$$
\begin{aligned}
N_{e}=\frac{P_{K} V_{a}}{\eta_{t r}}=\frac{m_{a} V_{a}}{\eta_{t r}} & {\left[g f+\frac{k F}{m_{a}} V_{a}^{2}+\frac{R^{2}+b^{2}+i_{Z}^{2}+f b\left(h-r_{d}\right)}{R} \times\right.} \\
\times & \left.\frac{d \omega_{Z}}{d t}+\omega_{Z}^{2} \cdot f \cdot\left(h-r_{d}\right)\right] .
\end{aligned}
$$

Analysis of the control structure (10) shows that the component of the engine power is

$$
N_{e_{1}}=\frac{m_{a} \cdot V_{a}}{\eta_{t r}} \cdot\left(g \cdot f+\frac{k \cdot F}{m_{a}} \cdot V_{a}^{2}\right) .
$$

It is used to ensure the steady rectilinear movement of the car, and the additional component of power

$$
N_{e_{2}}=\frac{m_{a} \cdot V_{a}}{\eta_{t r}} \cdot f \cdot\left(h-r_{d}\right) \cdot \omega_{Z}^{2}
$$

is used to ensure a steady cornering of the car with angular velocity of $\omega_{z}$ and the linear speed of $V_{a}$.

The power component

$$
N_{e_{3}}=\frac{m_{a} \cdot V_{a}}{\eta_{t r}} \cdot \frac{R^{2}+b^{2}+i_{Z}^{2}+f \cdot b \cdot\left(h-r_{d}\right)}{R} \cdot \frac{d \omega_{Z}}{d t}
$$

is the power necessary to maintain the created by steering the required steering action $\frac{d \omega_{Z}}{d t}$ when cornering.

Fig. 1 shows the definition of the realizable power of the engine during cornering. Modeling of the movement of a three-axle truck on a turn was carried out according to the method proposed in work [4].



Fig. 1: Determination of the realized power of the engine during cornering

The component of the engine power $N_{e_{1}}$, which is used to ensure a steady rectilinear motion of the car, is $16.07 \mathrm{~kW}$. The time dependence of the power component $N_{e_{2}}$ to ensure a steady rotation of the car is not more than $35 \mathrm{~W}$. The engine power expended on the movement of the car when turning, is not more than $180 \mathrm{~kW}$, while the maximum power developed by the engine of the car, according to the manufacturer, can reach $234 \mathrm{~kW}$.

At the initial movement along the straight line, the component $N_{e_{1}}$ does not depend on the driver's decision. It is determined by the total mass of the car, the rolling resistance coefficient $f$, the air resistance factor $k \cdot F$ and the specified driving speed (if the driving speed is set by external factors independent of the driver). The same reasoning is valid in case of a steady rotation of the car, in which $V_{a}$ and $\omega_{Z}$ are determined by the external conditions of motion.
The power component $N_{e_{3}}$ is the free power, redundant or control power, the value of which must be sufficient so that, given the turning radius $R$ and the linear speed $V_{a}$, the car could provide the angular acceleration $\frac{d \omega_{Z}}{d t}$ specified by steering. The component $N_{e_{3}}$ can be used as a reserve to ensure the stability of steady motion.

\section{Method for Building the Acceleration Characteristics of the Car Engine}

To estimate the dynamics of vehicle acceleration, the acceleration characteristic of the engine is shown in work [15], indicating the change in the effective power $N_{e}$ and the effective torque $M_{e}$ of the engine, depending on the angular velocity of the crankshaft during acceleration of the car.

The effective torque of the engine is determined by the following relationship [15]

$$
\begin{gathered}
M_{e}=\frac{m_{a} \cdot g \cdot r_{d}}{\eta_{t r} u_{t r}} \cdot\left(\psi+K_{t r} \cdot \varphi \cdot\left(\frac{S_{x}}{1-S_{x}}+\delta\right)-\right. \\
\left.-\frac{k \cdot F}{m_{a} \cdot g} \cdot\left(1-S_{x}\right)^{2} \cdot(\delta-1) \cdot \frac{n^{2}}{u_{t r}^{2}} \cdot r_{d}^{2}\right),
\end{gathered}
$$

where $u_{m p}$ is the total transmission ratio;

$\eta_{t r}$ is the transmission efficiency;

$r_{d}$ is the dynamic radius of the wheel;

$m_{a}$ is the gross vehicle weight; $g$ is acceleration of gravity;

$\varphi$ is the coefficient of traction of wheels with the road;

$K_{t r}$ is the coefficient of coupling weight use;

$\mathrm{k} \cdot \mathrm{F}$ is the air resistance factor;

$\delta$ is the coefficient of accounting for rotating masses of the engine and transmission;

$S_{x}$ is relative slipping;

$n$ is the angular velocity of the engine crankshaft;

$\psi$ is the total coefficient of road resistance.

Effective engine power [15]

$$
\begin{aligned}
N_{e}= & \frac{m_{a} \cdot g \cdot r_{d}}{\eta_{t r} \cdot u_{t r}}\left(\psi+K_{t r} \cdot \varphi\left(\frac{S_{x}}{1-S_{x}}+\delta\right)\right) n- \\
& -\frac{k \cdot F \cdot r_{d}{ }^{3} \cdot\left(1-S_{x}\right)^{2} \cdot(\delta-1)}{\eta_{t r} \cdot u_{t r}{ }^{3}} \cdot n^{3} .
\end{aligned}
$$

Expressions (14) and (15) do not take into account the change in the coefficient of adhesion depending on the linear speed of the car. In work [5], a number of approximating dependences for the determination of $\varphi(\mathrm{V})$ are proposed; some of them are given in Table. 1. One can take into account the dependence of $\varphi(\mathrm{V})$ in the construction of the engine acceleration characteristics by means of the following expression

$$
\begin{gathered}
N_{e}=\frac{m_{a} \cdot g \cdot r_{d}}{\eta_{t r} \cdot u_{t r}}\left(\psi+K_{t r} \cdot \varphi(V)\left(\frac{S_{x}}{1-S_{x}}+\delta\right)\right) n- \\
-\frac{k \cdot F \cdot r_{d}^{3} \cdot\left(1-S_{x}\right)^{2} \cdot(\delta-1)}{\eta_{t r} \cdot u_{t r}{ }^{3}} \cdot n^{3} .
\end{gathered}
$$

To build the acceleration characteristics of the car engine, it is necessary to know the value of the coupling weight utilization factor $K_{t r}$. 
Table 1: Results of approximation of the experimental dependence of the traction coefficient of wheels with the road $\varphi$ on the speed of the vehicle $V$ in $\mathrm{km} / \mathrm{h}$ and $\mathrm{m} / \mathrm{s}^{2}$

\begin{tabular}{|c|c|c|}
\hline & $\begin{array}{c}\text { Power-law model } \\
(V, \mathrm{~km} / \mathrm{h})\end{array}$ & $\begin{array}{c}\text { Power-law model } \\
(V, \mathrm{~m} / \mathrm{s})\end{array}$ \\
\hline$\varphi_{d r y}^{\max }(V)$ & $\begin{array}{c}0.1449+ \\
+0.9953 \cdot V^{-0.113}\end{array}$ & $\begin{array}{c}0.1386+ \\
+0.8672 \cdot V^{-0.112}\end{array}$ \\
\hline$\varphi_{d r y}^{\min }(V)$ & $0.8494-$ & $0.8494-$ \\
$\varphi_{\text {wet }}^{\max }(V)$ & $-0.0312 \cdot V^{0.616}$ & $-0.0687 \cdot V^{0.616}$ \\
\hline$\varphi_{\text {min }}(V)$ & $0.9865-$ & $0.9865-$ \\
\hline
\end{tabular}

For an all-wheel drive vehicle with the implementation of the limiting tangential reactions on the driving wheels $K_{t r}=1$, and for rear-wheel and front-wheel drive vehicles, respectively

$$
K_{t r} \cong \frac{a}{L-\delta \cdot \varphi \cdot h} ; K_{t r} \cong \frac{b}{L+\delta \cdot \varphi \cdot h} .
$$

Finally, the expressions for constructing the refined characteristics of the car engine will take the form of:

- for a rear-wheel drive car

$$
\begin{aligned}
& N_{e}=\frac{m_{a} \cdot g \cdot r_{\partial}}{\eta_{t r} \cdot u_{t r}} \cdot\left[\psi+\frac{a}{L-\delta \cdot \varphi \cdot h} \cdot \varphi(V) \times\right. \\
& \left.\times\left(\frac{S_{x}}{1-S_{x}}+\delta\right)\right] n-\frac{k F r_{d}{ }^{3}\left(1-S_{x}\right)^{2}(\delta-1)}{\eta_{t r} \cdot u_{t r}{ }^{3}} n^{3} ;
\end{aligned}
$$

- for a front-wheel drive vehicle

$$
\begin{aligned}
& N_{e}=\frac{m_{a} \cdot g \cdot r_{d}}{\eta_{t r} \cdot u_{t r}} \cdot\left[\psi+\frac{b}{L+\delta \cdot \varphi \cdot h} \cdot \varphi(V) \times\right. \\
& \left.\times\left(\frac{S_{x}}{1-S_{x}}+\delta\right)\right] n-\frac{k F r_{d}^{3}\left(1-S_{x}\right)^{2}(\delta-1)}{\eta_{t r} \cdot u_{t r}{ }^{3}} n^{3} ;
\end{aligned}
$$

- for a 4WD vehicle

$$
\begin{aligned}
N_{e} & =\frac{m_{a} \cdot g \cdot r_{d}}{\eta_{t r} \cdot u_{t r}}\left(\psi+\varphi(V)\left(\frac{S_{x}}{1-S_{x}}+\delta\right)\right) n- \\
& -\frac{k \cdot F \cdot r_{d}{ }^{3} \cdot\left(1-S_{x}\right)^{2} \cdot(\delta-1)}{\eta_{t r} \cdot u_{t r}{ }^{3}} \cdot n^{3} .
\end{aligned}
$$

\section{Experimental Evaluation of the Car Engine Performance}

The V-tech VT-2 loading dynamometer was used as a measuring equipment. Cars Opel Vectra and Mercedes C200 were used as objects of the study. The main characteristics of VT-2 are summarized in Table 2.

At the first stage of experimental research, four measurements of the engine characteristics of the Opel Vectra car - two with supercharging and two without supercharging were made. The maximum values of the effective power $N_{e} \max$ and the engine shaft rotation speed at the maximum power $\mathrm{n}_{\mathrm{N}}$ were recorded. Based on the measurement results, the discrepancy between the values of the maximum power $\Delta N_{e} \max$ and the values of the maximum torque $\Delta M_{e}$ max was determined.
Table 2: Characteristics of the used load dynamometer VT-2

\begin{tabular}{|l|c|}
\hline \multicolumn{1}{|c|}{ Parameter } & Value of parameter \\
\hline Dimensions (length $\times$ width), $\mathrm{mm}$ & $1300 \times 3500$ \\
\hline Maximum pressure per axle, $\mathrm{kg} 3000$ & 3000 \\
\hline Number of axles, pcs. & Telma $1 \times 350$ \\
\hline Brake, power, $\mathrm{kW}$ & 323,660 \\
\hline Dimensions of rotating shafts, $\mathrm{mm}$ & 300 \\
\hline Maximum speed on shafts, $\mathrm{km} / \mathrm{h}$ & 6000 \\
\hline Maximum thrust force on wheels, $\mathrm{H}$ & 0.25 \\
\hline $\begin{array}{l}\text { Accuracy of power measurement in the inertial } \\
\text { mode, } \%\end{array}$ & $\pm 1,0$ \\
\hline $\begin{array}{l}\text { Accuracy of power measurement applying the } \\
\text { brake, } \%\end{array}$ & 1350 \\
\hline kerb weight, $\mathrm{kg}$ & $\mathrm{f}$ \\
\hline
\end{tabular}

At the second stage of the experimental studies, four measurements of the characteristics of the engine of Mercedes C200 were performed.

The determination of engine characteristics was carried out in accordance with the requirements of international normative documents DIN 70020, EWG 80/1269, ISO 1585, JIS D1001 and SAE J1349.


Fig. 2: Installation of the vehicle on the load dynamometer VT-2: $\mathrm{a}$ - cooling the car engine during the tests; $\mathrm{b}$ - the method of fastening the car; $\mathrm{c}$ - fixing the front-wheel-drive vehicle to the foundation

The values of pressure and ambient temperature during the tests were taken according to the VT-2 stand sensors. The Telma brake was used to simulate road tests (in particular, when simulating air resistance while driving, as well as raising and lowering the car from slopes up to $45^{\circ}$ ). Before the measurements, the condition of the tires and the pressure in the wheels were checked.

To prevent the vehicle from coming down from the stand during the tests, its anchoring to the foundation was used with the help of hooks, belts and fastening clamps (see Fig. 2b, c). To simulate the airflow of the car with an oncoming airflow, a fan of $5.5 \mathrm{~kW}$ is used, creating a flow with a flow rate of $9360 \mathrm{~m}^{3} / \mathrm{h}$ (see Fig. 2a). To take readings during testing, they used software, fixing 2500 values per second. After the launch of the software, the status of the devices connected to the stand was determined. In the process of testing, the wheels of the car were accelerated before the transmission was engaged on which the measurements were to betaken, then the gas pedal was pushed to the stop, allowing the engine to gain maximum revolutions.

Then the clutch pedal was depressed, while releasing the acceleration pedal and waiting until the car wheels stopped. For testing cars without a turbocharger the inertial braking mode was used, and with the turbocharger the braking mode with loading was used. The results of bench tests on example of the engine performance of the Opel Vectra car are shown in Table 3. 
Table 3: Results of the bench test of the Opel Vectra car

\begin{tabular}{|c|c|c|c|c|c|c|}
\hline Metering & Boost & $N_{\text {e max }}, \mathrm{h}, \mathrm{p}$. & $N_{\text {e } \max }, \mathrm{kW}$ & $n_{N}, \mathrm{rev} / \min$ & $M_{\text {e } \max }, \mathrm{H} \cdot \mathrm{M}$ & $\begin{array}{c}n_{M}, \\
\mathrm{rev} / \mathrm{min}\end{array}$ \\
\hline 1 & - & 279.5 & 205.6 & 6758 & 291.4 & 6716 \\
\hline 2 & - & 287.8 & 211.7 & 7000 & 289.6 & 6903 \\
\hline 3 & + & 368.8 & 271.3 & 6521 & 425.3 & 4951 \\
\hline 4 & + & 325.3 & 239.3 & 5994 & 427.9 & 4979 \\
\hline
\end{tabular}

Analysis of Table 3 shows that according to the results of bench tests, the difference $\Delta N_{e \max }$ does not exceed $2.9 \%$; and $\Delta M_{e \max }$ does not exceed $0.6 \%$.

To assess the adequacy of the proposed method, let's compare the theoretical characteristics of the Opel Vectra car engine with the experimental $N_{\text {ebench }}(n)$. As the theoretical characteristics, we use the refined acceleration characteristics $N_{\text {e accel }}(n)$ and the external velocity characteristic of the engine $N_{\text {eescm }}(n)$. The results of the comparison are shown in Fig. 3.

$N_{e}$,

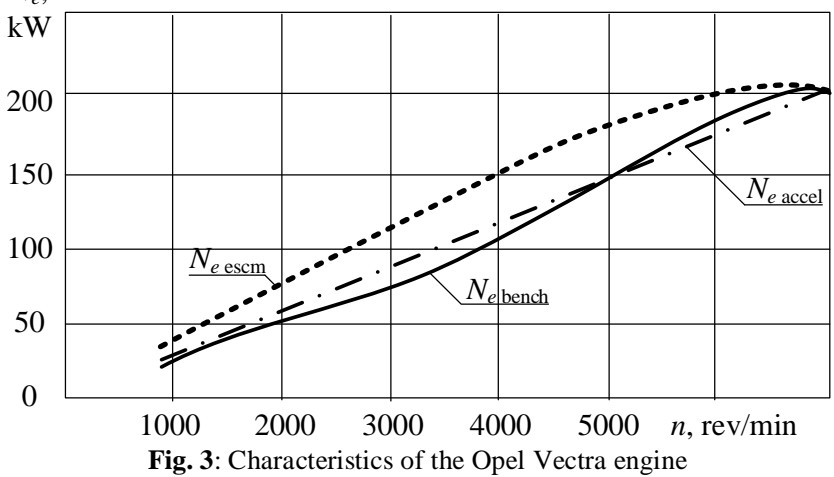

Similarly, the theoretical and experimental characteristics of the engine of the Mercedes C200 car are compared.

\section{Discussion of the Results of Study of Power Characteristics of the Car Engine}

The volume of free power (engine power reserve) allows the driver to implement the steering parameters, the position and speed of the steering wheel rotation, the turning parameters and the time of its performance.

Analysis of the results of experimental studies shows that the maximum discrepancy between $N_{\text {e escm }}$ and $N_{\text {e accel }}$ is $31 \pm 1 \%$, and the difference $N_{e \text { bench }}$ and $N_{\text {eaccel }}$ does not exceed $11 \pm 1 \%$. This indicates a significant reduction of the error in the theoretical evaluation of the effective power of the car engine with the help of acceleration characteristics in comparison with the traditional method of constructing the external high-speed characteristic of the engine.

\section{Conclusions}

The engine power reserve is necessary to provide the required maneuverability indicators. The engine power during maneuvering is spent on overcoming the resistance to movement and on providing the impact controlled by the maneuver. The first component of the engine power can be called a connected one, and the second can be called the free one or a control component. The received analytical expressions allow carrying out at a design stage a rational choice of the engine capacity by a condition of maintenance of demanded properties of maneuverability.

The developed method for constructing the refined acceleration characteristics of the engine makes it possible to determine the dynamic capabilities of the car, taking into account its design features and operating conditions.

When performing bench tests, the discrepancy between the maximum power values does not exceed $2.9 \%$, and the maximum torque values do not exceed $0.6 \%$. The maximum discrepancy between the values of the engine power, determined experimentally and using the classic traction calculation, is $31 \pm 1 \%$. The error obtained in the construction of the acceleration characteristics does not exceed $11 \pm 1 \%$. This indicates the adequacy of the proposed method of theoretical evaluation of the effective power of the car engine.

\section{Acknowledgement}

The authors are thankful to University of Technology (Baghdad, Iraq), Kharkiv National Automobile and Highway University (Kharkiv, Ukraine), National Technical University "Kharkiv Polytechnic Institute" (Kharkiv, Ukraine) and National Aerospace University "Kharkiv Aviation Institute" (Kharkiv, Ukraine) scientists for their cooperation in completing this study.

\section{References}

[1] Sinha A, Biswas A, Sharma K, "Comparison of efficiency of a solar driven carnot engine under maximum power and power density conditions", Journal of Urban and Environmental Engineering, Vol.8, No.2, (2014), pp. 225-231

[2] Turenko A, Podrygalo M, Klets D, Hatsko V \& Barun M, "A method of evaluating vehicle controllability according to the dynamic factor", Eastern-European Journal Of Enterprise Technologies, Vol.3, No.7(81), (2016), pp. 29-33.

[3] Mollenhauer K, Handbook of Diesel Engines, Springer-Verlag, Berlin Heidelberg, (2010), pp.15-16.

[4] Podrigalo NM, Pletnov VM, Abdulgazis UA, Abdulgazis AU, Polyanskiy OS, Podrigalo MA, (2012). The method for determining the car effective torque and effective engine power. Patent of Ukraine for useful model. G01L3/24. № 69235; declared 28.09.2011; published 25.04.2012, No.8.

[5] Podrigalo MA, Artiomov MP, "Algorithms of experimental determination of mobile machine engine power, which is realized when performing technological operations", Automobile- and tractor construction, Vol.60(966), (2012), pp.17-24.

[6] Idan A, Akimov O \& Kostyk K, "Development of a combined technology for hardening the surface layer of steel $38 \mathrm{Cr} 2 \mathrm{MoAl}$ ", Eastern-European Journal Of Enterprise Technologies, Vol.2, No.11(86), (2017), pp.56-62.

[7] Idan A, Akimov O, Golovko L, Goncharuk O \& Kostyk K, "The study of the influence of laser hardening conditions on the change in properties of steels", Eastern-European Journal Of Enterprise Technologies, Vol.2, No.5(80), (2016), pp.69-73.

[8] Chaoying X, Zhiming D, Cong Z, "A Single-Degree-of-Freedom Energy Optimization Strategy for Power-Split Hybrid Electric Vehicles", Energies, Vol. 10(7), (2017), p.23.

[9] Hannah ED, Brian PG, "Modelling future private car energy demand in Ireland", Energy Policy, Vol.39(12), (2011), pp.7815-7824.

[10] Fangjie Yu, Zhao L, "Direct Energy Rebound Effect of Family Cars: An Analysis Based on a Survey in Chang-Zhu-Tan City Group", Energy Procedia, Vol.104, (2016), pp.197-202.

[11] Karpievich Yu, Bakhanovich AG, Bondarenko II, "On-Board Monitoring of Technical State for Power Units of Wheeled and Tracked Vehicles", Nauka i Tehnika, Vol.5, (2016), pp.427-434.

[12] Wahono B, Xaoli W, Ogai H, "Combustion Property Analysis and Control System for the Dynamics of a Single Cylinder Diesel Engine", Mechatronics, Electrical Power, and Vehicular Technology, Vol.12, (2013), pp.117-126.

[13] Aliehyaei M, Atabi F, Khorshidvand M, Rosen M, "Energy, Economic and Environmental Analysis for Simple and Combined Heat and Power IC Engines", Sustainability, Vol.7, No.4, (2015), pp.4411-4424.

[14] Podrigalo M, Kaidalov R, Klets D, Podrigalo N, Makovetskyi A, Hatsko V, Abramov D, Tarasov Y, Lytovchenko D \& Litvinov A, "Synthesis of energy efficient acceleration control law of automobile", Eastern-European Journal Of Enterprise Technologies, Vol.1, No.7(91), (2018), pp.62-70.

[15] Podrigalo MA, Faist VL, "Accelerating characteristics of the automotive engine", Automobile- and tractor construction, Vol.58, (2008), pp.93-97. 